RU-92-58

\title{
Infinitely many regulator fields for chiral fermions
}

\author{
Rajamani Narayanan and Herbert Neuberger \\ Department of Physics and Astronomy \\ Rutgers University \\ Piscataway, NJ 08855-0849
}

\begin{abstract}
We show that two recent independent proposals for regularizing a chiral gauge theory stem from one common trick. If the anomaly free complex representation carried by the right handed fermi-fields is $r$ one constructs a vector like theory with flavored right handed fermionic matter in $r+\bar{r}$ but with a mass matrix of the order of the cutoff and having an index equal to unity in an infinite dimensional flavor space. We present a Pauli-Villars realization of the trick that is likely to work to all orders in perturbation theory and a lattice version which is argued to produce the correct continuum leading order fermionic contribution to the vacuum polarization tensor and readied for further perturbative checks.
\end{abstract}




\section{INTRODUCTION}

This paper addresses the problem of regularizing a nonabelian chiral gauge theory with right handed fermionic matter in an anomaly free complex representation of the gauge group. For definiteness we work in four dimensions and assume the model to be asymptotically free so that according to standard lore a continuum interacting theory with no free parameters exists. Following [1] we refer to this continuum theory as the target theory; a concrete example for a target theory is $S O(10)$ with righthanded fermions in the spinorial representation. We shall focus on a particular trick that opens up the possibility for a gauge invariant regularization at the expense of introducing an infinite set of fermionic regulator fields. Two recent independent proposals [2,3], although quite different in appearance, can be built around this trick.

We start in Minkowski space. The gauge group index will be suppressed and plays no role in most of what follows. To each righthanded fermion of the target theory we attach a tower consisting of righthanded and lefthanded fermionic fields labeled by a new index that lives in a new internal space. The total fermion number of each tower is conserved.

We wish to give Dirac masses of the order of the cutoff to all the extra fields and, at the same time, make the theory look vector like and gauge invariant. We write the Lagrangian density as:

$$
\begin{aligned}
\mathcal{L} & =\mathcal{L}_{1}+\mathcal{L}_{g}(A), \quad \mathcal{L}_{1}=i \bar{\psi}(\not \partial-i g A) \psi+\bar{\psi}\left(M P_{R}+M^{\dagger} P_{L}\right) \psi \\
P_{R} & =\frac{1+\gamma_{5}}{2}, \quad P_{L}=\frac{1-\gamma_{5}}{2}, \quad A_{\mu}=A_{\mu}^{a} T^{a}, \quad \operatorname{tr}\left(T^{a} T^{b}\right)=\delta^{a b}
\end{aligned}
$$

To make the theory look vector like we need to identify the internal spaces associated with the lefthanded and righthanded fields and $M$ becomes an operator acting in this space. The free fermionic theory will have massless righthanded particles equal in number to $n_{R}=$ $\operatorname{dim}(\operatorname{Ker}(M))$ and massless lefthanded particles equal in number to $n_{L}=\operatorname{dim}\left(\operatorname{Ker}\left(M^{\dagger}\right)\right)$. If the internal space has a finite dimension, $n_{R}=n_{L}$, and we cannot get to the target theory. To make $n_{R}=1$ and $n_{L}=0$ we need an infinite dimensional space; a simple example is $M=\Lambda \mathbf{a}$ where $\Lambda$ is the cutoff and $\mathbf{a}$ is a bosonic annihilation operator.

If $M$ were a finite matrix and $M^{\prime}$ its restriction to the subspace orthogonal to $\operatorname{Ker}(M)$ the parity breaking appearance of eq. (1) could be eliminated by $\psi \rightarrow \exp \left(i \alpha \gamma_{5}\right) \psi$ where $\alpha$ is a hermitian matrix satisfying $\exp (2 i \alpha)=\left(M^{\prime} M^{\prime \dagger}\right)^{-1 / 2} M^{\prime}$. This doesn't work in our case and parity is not conserved. However, one can define $U=M^{\dagger}\left(M M^{\dagger}\right)^{-1 / 2}$ and if one "forgets" that $U$ is only formally unitary* the theory looks vector like and easy to regularize.

$* \overline{U^{\dagger} U=1 \text { but } U U^{\dagger}=M^{\dagger}}\left(M M^{\dagger}\right)^{-1} M=1-Q$ where $Q$ is the projector on the zero eigenspace of $M$. 
One can easily find more examples of $M$ but one should try to keep the structure as simple as possible. Before we had $M / \Lambda=\exp \left(-\frac{s^{2}}{2}\right) \partial_{s} \exp \left(\frac{s^{2}}{2}\right)$ where $s \in \mathrm{R}$ is the internal space "coordinate" of the harmonic oscillator. This can be generalized to $M / \Lambda=$ $\exp (-F(s)) \partial_{s} \exp (F(s))$ where $\exp (-F(s))$ is square integrable and the internal space is the space of square integrable functions on the line. This choice assures that the kernels of $M$ and its adjoint will have the correct dimensions. One can also generalize to an internal space corresponding to a complex coordinate with $M / \Lambda=\exp (-F(z, \bar{z})) \partial_{z} \exp (F(z, \bar{z}))$. In the last example $M$ represents one chirality component of a Dirac particle living in two Euclidean dimensions in an external magnetic field and the flux of that field must be appropriately adjusted to get the correct number of zero modes. The other chirality component is represented by $M^{\dagger}$.

In all the examples above the internal space corresponds to additional internal dimensions and ultraviolet behavior might be worse than in the bare theory because there are more single particle states at high energies. Therefore, regularization has to deal with all the original divergences and also potential new ones. If the spectrum of $M M^{\dagger}$ is discrete the fields can be labeled by $0,1,2, \ldots$ and it is natural to view the extra fields as Pauli-Villars regulator fields whose masses and statistics are adjusted so that everything is ultraviolet finite to any order in perturbation theory. If the spectrum of $M M^{\dagger}$ has an unbounded continuous component it is more natural to first try to get rid of the extra ultraviolet divergences induced by the internal space and this is easiest done by latticizing the variable $s$ and thereby bounding the spectrum of $M M^{\dagger}$ from above. It is natural then to also latticize real space and try to get a non-perturbative definition of the target theory with hopes of addressing such long standing vexing basic questions about chiral gauge theories as, for example, what their spectrum is. We explore both possibilities in this paper.

We first analyze the Pauli-Villars case and show that it essentially corresponds to the suggestion of [2]. Our point of view seems quite different from the one adopted there, to the extent we understand it; our final formulae can be made to differ only in some of the details and we think that the differences are insignificant. The indications are that the method works for an anomaly free situation but breaks down when the gauge group is represented by fermions in an anomalous representation. Ungauged, global nonabelian symmetries can be preserved only if they are non-anomalous. Next we discuss the lattice case and show that it is closely related to the proposal of [3]. Our version will differ from this proposal in the manner in which the fermions are coupled to the gauge fields; we shall not introduce extra gauge or other bosonic fields beyond those present in the target theory. Our study will be somewhat preliminary: we shall provide a necessary tool that was missing until now which is an explicit formula for the fermionic propagator and shall argue that the vacuum polarization induced by fermions has the right continuum limit. We hope to come back in the future with a more detailed perturbative study of the lattice 
version; as far as we can see now the outlook for this approach is better than anything we have seen in years, but a completely well defined construction is still lacking and more work is needed.

\section{PAULI-VILLARS}

We assume that $M M^{\dagger}$ and $M^{\dagger} M$ have discrete nondegenerate spectra. In addition we assume the existence of a "parity" operator $\hat{S}$ which anticommutes with $M$, is hermitian and squares to unity. The pure gauge part is regularized in some way, unspecified except that it preserves ordinary, continuous, space-time structure and gauge invariance. For example, a higher derivative regularization, as defined in [4], does this. We are considering in this subsection only perturbation theory and any loop with at least a gauge or ghost internal line is assumed to be made finite by the extra regularization. Thus, all we need to do is to construct a gauge invariant regularization of the fermionic determinant in an arbitrary external gauge field. Not surprisingly, the overall regularization scheme will only work if the representation of the gauge group carried by the righthanded fermions is anomaly free.

The free fermion propagator is:

$$
\left[\not p-M P_{R}-M^{\dagger} P_{L}\right]^{-1}=\left(\not p+M^{\dagger}\right) P_{R} \frac{1}{p^{2}-M M^{\dagger}}+(\not p+M) P_{L} \frac{1}{p^{2}-M^{\dagger} M}
$$

We choose the statistics to be well defined for fields that are eigenstates of $\hat{S} \gamma_{5}$. In this way the Lagrangian only couples fields of the same statistics: the $\not \partial-A$ term is unity in internal space and couples only fields of the same handedness while the mass terms only couple fields of opposite handedness and opposite $\hat{S}$ parity. A fermion loop is given by tracing over internal space, spinor index, gauge group index and integrating over the loop momentum. Statistics is taken into account by inserting at some point one statistics factor

equal to $-S$. To get the right target theory one needs to pick the $\hat{S}$ parity of the zero eigenvector of $M$ as unity.

As an example consider the lowest order contribution to the vacuum polarization, given by $\left\langle j_{\mu, a} j_{\nu, b}\right\rangle=\delta_{a b} \Pi_{\mu \nu}$. After a certain amount of algebra one obtains:

$$
\Pi_{\mu \nu}(p)=\int \frac{d^{4} k}{(2 \pi)^{4}}\left[\operatorname{Tr}\left[\hat{S}\left(1-\frac{Q}{2}\right) \frac{1}{q_{+}-\sqrt{M^{\dagger} M}} \gamma_{\mu} \frac{1}{q_{-}-\sqrt{M^{\dagger} M}} \gamma_{\nu}\right]+\frac{1}{2} \operatorname{tr}\left[\frac{1}{d_{+}} \gamma_{\mu} \frac{1}{d_{-}} \gamma_{\nu} \gamma_{5}\right]\right]
$$

$q_{ \pm}=k \pm \frac{p}{2}$ and $\operatorname{Tr}$ denotes a trace over everything while $t r$ sums over spinor indices.

The $\gamma$ matrix trace in the last term is proportional to $\epsilon_{\mu \nu \alpha \beta} q_{+}^{\alpha} q_{-}^{\beta}$; under $k \rightarrow-k$ the term is odd and hence the momentum integral over it vanishes. This formal argument*

* The last term in eq. (3) does not converge by power counting. 
can be easily made rigorous. Once the first term in eq. (3) is arranged to be finite the transversality of the vacuum polarization tensor will be assured by the usual (no longer formal) argument.

To understand finiteness let us consider a slightly more general type of loop integral $\int \frac{d^{4} k}{(2 \pi)^{4}} I(k)$ with

$$
I(k)=\operatorname{polynomial}\left(q_{j}\right) \operatorname{Tr}\left[\hat{S} \prod_{j=1}^{n}\left(\frac{1}{q_{j}^{2}-M^{\dagger} M}\right)\left(1-\frac{Q}{2}\right)\right]
$$

where $q_{j}=q_{j-1}+p_{j}, \quad j=2,3, \ldots . n \quad q_{1}=k$ and the $p_{j}$ are external momenta that sum to zero. The trace is over the internal space. Pick the basis of the space as $|0\rangle,|E\rangle$ with $M^{\dagger} M \mid 0>=0$ and $M^{\dagger} M|E>=E| E>, E>0$. We assume that there are no degeneracies. Since $\hat{S}$ commutes with $M^{\dagger} M$ we have $\hat{S}\left|E>=\eta_{E}\right| E>, \hat{S}|0>=| 0>$ and $\eta_{E}= \pm 1$. After exponentiating each of the denominators with the help of a parameter $t_{j}$ and rotating to Euclidean space it is clear that the question of convergence is settled by the behavior of the trace when all $t_{j}$ 's are scaled to zero. Let $\sum t_{j}=\tau$; we have to consider then the behavior of

$$
S u m=2 \sum_{E} \eta_{E} \exp (-E \tau)+1
$$

Because of gauge invariance and absence of anomalies we only need to tame the logarithmic divergence of a few diagrams. It is then sufficient to make the expression in eq. (5) vanish linearly when $\tau$ goes to zero. This is easily achieved with $M=\Lambda \mathbf{a}$ and $\hat{S}=(-1)^{\mathbf{a}^{\dagger} \mathbf{a}}$ leading to $S u m=\tanh \left(\Lambda^{2} \tau\right)$. Better control in the ultraviolet is achievable; for example one may be interested in the determinant of $\not \partial-A$ in higher even dimensions. An arbitrary degree of divergence can be tamed by choosing $M=\Lambda \sqrt{\mathbf{a a}^{\dagger}} \mathbf{a}$ and the same statistics operator as above. Now the sum becomes $\theta_{4}\left(0 \mid e^{-\Lambda^{2} \tau}\right)$ and the Jacobi identity ensures that it vanishes faster than any power when $\tau$ approaches zero. This latter choice has been made in [2].

At any order we shall have to deal with one extra piece, similar to the one we discarded in the calculation of the vacuum polarization, before we can restrict our attention to sums of the kind shown in eq. (4). At higher orders these extra terms converge by power counting but diagrams with three and four vertices may be divergent and untamable. Our investigations indicate that if the theory is anomaly free no problems are encountered and everything goes through as for the vacuum polarization graph.

Essentially, the Pauli-Villars regularization removes all divergences in the parity conserving part of the fermionic determinant but does not touch the parity odd part of the logarithm of this determinant. The latter must be finite and unambiguous "by itself" without any help from the Pauli-Villars regulator fields and this happens only in the anomaly free case. 
It is important to make sure that the counter terms will not affect the zero mode structure of the mass matrices; otherwise the target theory would not be attained. This is easily checked to leading order and turns just into a matrix generalization of the well known $\delta m_{f} \propto m_{f}$ rule. Here this works is an obvious way because the internal space is totally decoupled from the real space and the spinor and group indices. However, the mechanism that protects the desired zero mode structure from radiative corrections is a deeper one and this becomes an essential feature when we turn to the lattice where such a decoupling cannot be achieved because of the well known "lattice fermion doubling". The lattice version therefore also avoids infinite amount of fermion mass fine tuning [5].

\section{LATTICE}

A simple choice of the mass matrix in the lattice case is based on the "wall" realization of $M$. Its continuum form is $M / \Lambda=\partial_{s}+f(s), f(s)=F^{\prime}(s)$ with $f$ increasing monotonically from a negative asymptotic value to a positive one. In particular, this means that the internal manifold is not compact. $M$ will have a zero eigenfunction and $M^{\dagger}$ will have none; this feature is stable under deformations that do not change the large $|s|$ behavior [6]: $M M^{\dagger}$ and $M^{\dagger} M$ have identical spectra for nonvanishing eigenvalues and therefore any small deformation of $M$ cannot move the zero mode of $M^{\dagger} M$ up without pairing it up with an eigenstate of $M M^{\dagger}$ of exactly the same energy; however we assume that there is a finite gap between the zero mode and the rest of the spectrum and a small deformation cannot drag down an eigenstate of $M M^{\dagger}$ far enough. It is hoped that radiative corrections will be well behaved in this sense if the theory is anomaly free. Plugging the "wall" form of $M$ into eq. (1) leads to the interpretation of $s$ as a fifth dimension, the derivative part of $M$ and $M^{\dagger}$ (i.e. $\frac{M-M^{\dagger}}{2}$ ) combining with the kinetic energy into a five dimensional Dirac operator, and the $f(s)$ dependent part (i. e. $\frac{M+M^{\dagger}}{2}$ ) becoming a variable mass term.

The spectrum of $M M^{\dagger}$ is unbounded and to cure this we replace inner space by an infinite lattice in both directions. $s$ now denotes the discrete integer labeling the sites on this internal lattice and $M$ is replaced by a first order difference operator: $M_{s, s^{\prime}}=$ $\delta_{s+1, s^{\prime}}-a(s) \delta_{s, s^{\prime}}$. If $a(s)$ approaches $a_{+}$with $\left|a_{+}\right|<1$ when $s \rightarrow \infty$ and $a_{-}$with $\left|a_{-}\right|>1$ when $s \rightarrow-\infty$ the spectral properties we need are preserved. For definiteness we choose $a^{0}(s)=1-m_{0} \operatorname{sign}\left(s+\frac{1}{2}\right)$ with $0<m_{0}<1$ and denote the corresponding $M$ by $M^{0}$.

Putting an Euclidean version (with hermitian $\gamma$ matrices) of eq. (1) on the lattice we 
obtain:

$$
\begin{aligned}
-\mathcal{L}_{E}= & -\beta \sum_{x, \mu} \Re\left\{\operatorname{tr}\left[U_{\mu}(x) U_{\nu}(x+\mu) U_{\mu}^{\dagger}(x+\nu) U_{\nu}^{\dagger}(x)\right]\right\}+\sum_{x, \mu} \frac{1}{2} \bar{\psi}(x) \gamma_{\mu}\left[U_{\mu}(x) \psi(x+\mu)\right. \\
& \left.-U_{\mu}^{\dagger}(x-\mu) \psi(x-\mu)\right]+\sum_{x} \bar{\psi}(x)\left[P_{R}\left(M\left(\nabla_{U}\right) \psi\right)(x)+P_{L}\left(M^{\dagger}\left(\nabla_{U}\right) \psi\right)(x)\right] \\
& \left(\nabla_{U} \psi\right)(x)=\sum_{\mu}\left[U_{\mu}(x) \psi(x+\mu)+U_{\mu}^{\dagger}(x-\mu) \psi(x-\mu)-2 \psi(x)\right]
\end{aligned}
$$

For noninteracting fermion fields in Fourier space, $M$ depends parametrically on the lattice momentum $p\left(-\pi<p_{\mu} \leq \pi\right)$ with $\nabla_{U=1} \equiv \nabla=-4 \sum_{\mu} \sin ^{2} \frac{p_{\mu}}{2}$ so that all the unwanted potential zeros of the propagator are moved up in energy by Wilson mass terms. We have to require that for $\nabla$ in a region close to $p_{\mu}=0 \operatorname{dim}(\operatorname{Ker}(M(\nabla)))=1$ but for $\nabla \leq-4$ $\operatorname{dim}(\operatorname{Ker}(M(\nabla)))=0$. We also require that $M^{\dagger}$ have no zero modes in regions around the momenta $p$ with $\sin \left(p_{\mu}\right)=0$ for any $\mu$. We need to change the asymptotic behavior in $s$ so that as $\nabla$ varies over the Brillouin zone the spectra of $M(\nabla)$ and $M^{\dagger}(\nabla)$ change as follows: for small momenta that satisfy $-\nabla<h$ (an example for $h$ will be provided later), $M$ has a normalizable zero mode and the spectrum of $M(\nabla) M^{\dagger}(\nabla)$ is continuous with a finite gap separating it from zero. On the boundary of this small momentum region, $-\nabla=h$, the spectra of $M(\nabla) M^{\dagger}(\nabla)$ and $M^{\dagger}(\nabla) M(\nabla)$ are identical, continuous and gapless. Outside the region, where $-\nabla>h$, the spectra of $M(\nabla) M^{\dagger}(\nabla)$ and $M^{\dagger}(\nabla) M(\nabla)$ are identical, continuous and there is a gap. All unwanted "doubler modes" of the naïve fermionic action are in this third region. Note that the abrupt disappearance of the zero mode at the boundary between the two regions is a necessary consequence of the generic structure of the supersymmetric quantum mechanics governing the internal space and not of the specific choices we have made. In short, the trick in the lattice version amounts to walling a small region of momentum space around the origin off the dangerous areas inhabited by doublers. The spectrum has a nonanalyticity in momentum space as a result and one has to check carefully for effects caused by this*. Clearly, these nonanalyticities come about because internal space is infinite. The divergence responsible for the singularity on the four dimensional Fourier torus is an infrared problem from the point of view of the extra dimension. Other approaches $[7,8]$ that tried to use singularities fail because gauge invariance forces compensating singular changes in the gauge fermion vertices $[9,10,11]$ and these introduce unwanted doublers, ghosts, Lorentz violations or uncomputable nonperturbative effects $[11,12,13]$ into the theory. Somehow, this new trick has to avoid all of these traps.

We know that the zero modes of $M$ live in the vicinity of the wall in the five dimensional world and that the physics we are interested in is the defect dependent part of the

* $\overline{\text { Of course, some singularity }}$ is needed to avoid doublers in a non-dynamical way. 
action. To eliminate five dimensional bulk effects the effective gauge interaction induced by fermions, $S_{\text {eff }}(U, w a l l)$, is replaced by $S_{\text {eff }}(U)=S_{\text {eff }}(U$, wall $)-\frac{S_{\text {eff }}^{+}(U)+S_{\text {eff }}^{-}(U)}{2}$ where the $S_{\text {eff }}^{ \pm}(U)$ come from systems with constant mass terms equal to $\pm m_{0}$. The needed subtractions can also be interpreted as representing ghost fields. In analytical computations, both in weak and in strong coupling expansions, the needed subtractions should be implementable order by order.

We make our remaining choices so that the homogeneous systems which provide the subtractions be as symmetric as possible. We would like to have reflection positivity in the five dimensional system in all directions in the homogeneous case and five dimensional cubic symmetry when $U=1$. Clearly, the relevant five dimensional systems have the same Lagrangian as above, only the matrix $M^{0}$ gets replaced by $M^{ \pm}$with the corresponding $a^{0}(s)$ replaced by $a^{ \pm}(s)=1 \pm m_{0}$. The five dimensional symmetries we want are then obtained by making in each case $M\left(\nabla_{U}\right)=M^{\sigma}+\frac{\nabla_{U}}{2}$ with $\sigma=0, \pm$. It is easy to see that with this choice the origin of four dimensional momentum space is correctly walled off with $0<h=2 m_{0}<2$. Note that we have more or less naturally ended up in the $r=1$ case of the Wilson mass term (for notations see [14]); this case has better positivity properties than cases where $|r| \neq 1$ because it has a positive bounded transfer matrix for single step translations $[15,16,17]$ so we should feel no need to generalize $[3,18,19]$.

The candidates for observables in the target theory are obtained as follows: Pure gauge field operators are naïvely transcribed. Local four dimensional fermions are represented by a sum over $s$ of the bare fermion fields with a weight that samples a finite but wide region around $s=0$. The width of the weight functions should be taken to infinity at the very end. Composite operators of the target theory are best constructed directly in the regularized version, for example, the currents associated with global symmetries will have a sum over all $s$ in their definition via Noether's formula. With this definition the argument that correct chiral anomalies are obtained [3,19,20,21,22,23] applies. The contributions of the subtraction terms $S_{\text {eff }}^{ \pm}(U)$ to anomalies cancel against each other because of the opposite signs of the mass terms. One expects currents associated with anomalous global symmetries to be affected by the infinite sum over $s$ in a non-trivial way, enabling the charges to flow out to infinity and hence be nonconserved in any finite five dimensional slab containing the defect. This shows that is is unclear whether exact unitarity holds if one looks only at such arbitrarily large, but still finite, slabs. A proper construction will define the whole theory in such a slab with boundary conditions is the $s$ direction (the other directions are irrelevant and can be kept infinite or made finite by standard periodic/antiperiodic boundary conditions) chosen in such a way that anomalous charges will be able to flow out of the system. If the gauged group is not anomalous one would like then to see that a finite limit is obtained when the slab's thickness is taken to infinity. The choice of good boundary conditions needs care because in a finite dimensional internal 
space the zero modes will typically appear both in $M$ and in $M^{\dagger}$; this might be avoidable if the boundary conditions replace $M$ and $M^{\dagger}$ by matrices that are not each other's adjoint but this would give up the unitarity of the theory for any finite width of the slab.

The advantage of the present approach is that one can set up a meaningful test of the construction within perturbation theory and this test can be carried out before the issue of boundary conditions is settled since the subtractions remove large $s$ divergences and one can work always with an infinite internal space. As in the Pauli-Villars case we consider the fermion contribution to the vacuum polarization; this only tests the correctness of $S_{\text {eff }}(U)$ but is known to be a test failed by all previous proposals. From the imaginary part of the polarization tensor at small momenta one can read off the spectrum of fermions as seen by the gauge bosons and this is the relevant question. It is obvious that naïvely the method works because the propagator has the right structure at nearly zero momenta by construction. The only way this argument can fail in perturbation theory is that there are further singularities in the integrand of the relevant lattice Feynman diagrams and they make contributions to the continuum limit. If we think about the propagator in a basis of eigenstates of $M^{\dagger}(\nabla) M(\nabla)$ and $M(\nabla) M^{\dagger}(\nabla)$ there is a source of trouble as discussed before. We aim to show now that if one represents the propagators in four dimensional lattice Fourier space and in $s$-space one can perform the needed subtractions easily and no singularities beyond the "good" ones appear. In particular, none are induced by carrying out the $s$-space traces.

The propagator is given by a formula similar to eq. (2), now on the lattice and in Euclidean space. With $\bar{p}_{\mu}=\sin \left(p_{\mu}\right)$ and $\hat{p}_{\mu}=2 \sin \frac{p_{\mu}}{2}$ we have:

$$
\begin{gathered}
\left(-i \sum_{\mu} \gamma_{\mu} \bar{p}_{\mu}+M^{\dagger}(\hat{p})\right) P_{R} G_{R}(p)+\left(-i \sum_{\mu} \gamma_{\mu} \bar{p}_{\mu}+M(\hat{p})\right) P_{L} G_{L}(p) \\
G_{R}(p)=\frac{1}{\sum_{\mu} \bar{p}_{\mu}^{2}+M(\hat{p}) M^{\dagger}(\hat{p})}, \quad G_{L}(p)=\frac{1}{\sum_{\mu} \bar{p}_{\mu}^{2}+M^{\dagger}(\hat{p}) M(\hat{p})} \\
G_{R}(p) M(p)=M(p) G_{L}(p)
\end{gathered}
$$

$G_{R}(p)$ and $G_{L}(p)$ are inverses of second order difference operators and an explicit expression can be obtained by standard techniques. Both are symmetric under the interchange of $s$ and $s^{\prime}$ and for $s \geq s^{\prime}$ they are given by

$$
\begin{aligned}
& {\left[G_{R}\right]_{s s^{\prime}}(p)= \begin{cases}{\left[A_{R}(p)-B(p)\right] e^{-\alpha_{+}(p)\left[s+s^{\prime}+2\right]}+B(p) e^{-\alpha_{+}(p)\left[s-s^{\prime}\right]}} & \text { for } s^{\prime} \geq-1 \\
A_{R}(p) e^{-\alpha_{+}(p)[s+1]+\alpha_{-}(p)\left[s^{\prime}+1\right]} & \text { for } s \geq-1 \geq s^{\prime} \\
{\left[A_{R}(p)-C(p)\right] e^{-\alpha_{-}(p)\left[s+s^{\prime}+2\right]}+C(p) e^{-\alpha_{-}(p)\left[s-s^{\prime}\right]}} & \text { for } s \leq-1\end{cases} } \\
& {\left[G_{L}\right]_{s s^{\prime}}(p)= \begin{cases}{\left[A_{L}(p)-B(p)\right] e^{-\alpha_{+}(p)\left[s+s^{\prime}\right]}+B(p) e^{-\alpha_{+}(p)\left[s-s^{\prime}\right]}} & \text { for } s^{\prime} \geq 0 \\
A_{L}(p) e^{-\alpha_{+}(p) s+\alpha_{-}(p) s^{\prime}} & \text { for } s \geq 0 \geq s^{\prime} \\
{\left[A_{L}(p)-C(p)\right] e^{-\alpha_{-}(p)\left[s+s^{\prime}\right]}+C(p) e^{-\alpha_{-}(p)\left[s-s^{\prime}\right]}} & \text { for } s \leq 0\end{cases} }
\end{aligned}
$$


where

$$
\begin{gathered}
0 \leq \alpha_{ \pm}(p)=\operatorname{arccosh}\left\{\frac{1}{2}\left[a_{ \pm}(p)+\frac{1}{a_{ \pm}(p)}+\frac{\bar{p}^{2}}{a_{ \pm}(p)}\right]\right\}, \quad a_{ \pm}(p)=1 \mp m_{0}+\frac{1}{2} \hat{p}^{2} \\
A_{R}(p)=\frac{1}{a_{-}(p) e^{\alpha_{-}(p)}-a_{+}(p) e^{-\alpha_{+}(p)}}, \quad A_{L}(p)=\frac{1}{a_{+}(p) e^{\alpha_{+}(p)}-a_{-}(p) e^{-\alpha_{-}(p)}} \\
B(p)=\frac{1}{2 a_{+}(p) \sinh \alpha_{+}(p)}, \quad C(p)=\frac{1}{2 a_{-}(p) \sinh \alpha_{-}(p)}
\end{gathered}
$$

The propagator needed for the subtraction terms are obtained by replacing all $a_{ \pm}, \alpha_{ \pm}$by either $a_{+}, \alpha_{+}$or $a_{-}, \alpha_{-}$. Under each of these substitutions $A_{R}(p), A_{L}(p), B(p)$ and $C(p)$ all become equal to each other. It is easy to see that $\alpha_{ \pm}(p)>0$ for all $p$. Subtractions make the sum over $s, s^{\prime}$ converge exponentially and uniformly in the loop momentum $k$ and external momentum $p$. All ordinary manipulations that prove the transversality of $\Pi_{\mu \nu}(p)$ and its symmetry properties $\Pi_{\mu \nu}(p)=\Pi_{\nu \mu}(p)=\Pi_{\mu \nu}(-p)$ hold after the subtractions. All vertex factors are singularity free throughout the Brillouin zone. The only singularities in a typical integrand then come from the possible poles in the amplitudes $A_{R}(p), A_{L}(p), B(p)$ and $C(p)$. Since we have arranged for $M(\hat{p})$ to have a zero mode, there is a singularity at $p=0$ in $A_{L}(p)$. It is easy to see from the expressions for the amplitudes that this is the only singularity. A simple calculation gives

$$
A_{L}(p)=\frac{m_{0}\left(4-m_{0}^{2}\right)}{4 p^{2}}+\text { regular terms }
$$

The residue of the pole is just the normalization factor associated with the zero mode at $p=0$. The usual continuum expressions should therefore emerge. We end our perturbative analysis remarking that all ingredients for the calculation of $\Pi_{\mu \nu}(p)$ are explicitly present and a meticulous calculation will make the above argument foolproof.

We can also address the issue of how the approach presented here avoids the NielsenNinomiya [24] theorem. To this end, consider integrating all the fermion degrees of freedom except the righthanded fermion at $s=0$. The kinetic energy term will be non-local and can be extracted from the expression for the propagator at $s=s^{\prime}=0$. We find that the kinetic energy term is given by

$$
\frac{1}{A_{L}(p) \bar{p}^{2}} i \sum_{\mu}\left[\gamma_{\mu} \bar{p}_{\mu}\right] P_{R}
$$

By eq. (10) $A_{L}(p) \bar{p}^{2}$ goes to the normalization factor of the zero mode and eq. (11) has the expected zero at $p=0$. The factor $\bar{p}^{2}$ in the denominator generates fifteen poles in the Brillouin zone. Normally these poles would have generated ghost contributions [10] and destroyed the theory. But, in this approach, integrating out the fermion degrees 
of freedom also generates pure gauge terms and these cancel the contributions from the ghosts. In earlier attempts to solve the problem [8], it was understood that the bgauge part of the action has to be suitably adjusted to cancel the ghost contributions. The approach presented here can be thought of as one way to systematically achieve the apparently difficult aim faced before.

\section{FINAL COMMENTS}

It should be clear from this paper that there is a large amount of latitude in choosing $M$ and the associate internal space. It is not clear at the moment whether sticking to the "wall" situation is the best, but certainly it seems a worthwhile route to pursue, in particular because it gives a familiar picture for where the anomalous charge deficit is going. The basic trick however may be useful even if the "wall" realization turns out to

fail ultimately; in its essence what the trick does is to provide the "infinite hotel" that has been argued to be a necessity for the existence of genuinely chiral fermions [24].

\section{ACKNOWLEDGEMENTS}

This research was supported in part by the DOE under grant \# DE-FG05-90ER40559.

\section{REFERENCES}

1) A. Borrelli, L. Maiani, G. C. Rossi, R. Sisto, M. Testa, Nucl. Phys. B333 (1990) 335.

2) S. A. Frolov, A. A. Slavnov, SPhT/92-051, Saclay preprint, 1992.

3) D. B. Kaplan, Phys. Lett. B288 (1992) 342.

4) L. D. Faddeev, A. A. Slavnov, "Gauge Fields, an Introduction to Quantum Field Theory", Second Edition, Addison-Wesley, (1991).

5) A. C. Longhitano, B. Svetitsky, Phys. Lett. B126 (1983) 259.

6) E. Witten, Nucl. Phys. B185 (1981) 513. 
7) S. D. Drell, M. Weinstein, S. Yankielowicz, Phys. Rev. D14 (1976) 487.

8) C. Rebbi, Phys. Lett. B186 (1987) 200.

9) L. H. Karsten, J. Smit, Nucl. Phys. B144 (1978) 536.

10) A. Pelissetto, Ann. Phys. (N.Y.) 182 (1988) 177.

11) G. T. Bodwin, E. V. Kovacs, Phys. Rev. D35 (1987) 3198.

12) J. M. Rabin, Phys. Rev. D12 (1981) 3218.

13) M. Weinstein, Phys. Rev. D26 (1982) 839.

14) J. Smit, Acta Physica Polonica, B17 (1986) 531.

15) P. Menotti, A. Pelissetto, Nucl. Phys. B(Proc. Suppl.) 4 (1988) 644.

16) M. Creutz, Phys. Rev. D35 (1987) 1460.

17) M. Lüscher, Comm. Math. Phys. 54 (1977) 283.

18) K. Jansen, M. Schmaltz, UCSD/PTH 92-29, UCSD preprint, 1992.

19) M. F. L. Golterman, K. Jansen, D. B. Kaplan, UCSD/PTH 92-28, UCSD preprint, 1992.

20) C. G. Callan, Jr., J. A. Harvey, Nucl. Phys. B250 (1985) 427.

21) S. G. Naculich, Nucl. Phys. B296 (1988) 837.

22) A. Coste, M. Lüscher, Nucl. Phys. B323 (1989) 631.

23) K. Jansen, Phys. Lett. B288 (1992) 348.

24) H. B. Nielsen, M. Ninomiya, in "Trieste Conference on Topological Methods in Quantum Field Theories", World Scientific, 1991. 